\title{
Enabling Smarter Cities with Operations Management
}

\author{
Ho-Yin Mak \\ Saïd Business School, University of Oxford, ho-yin.mak@sbs.ox.ac.uk
}

\begin{abstract}
Recent development of new technologies and data analytics has unleashed the so-called smart city movement, which has been transforming the ways that cities (and their services) operate. This movement is in line with the Operations Management (OM) community's pursuit of innovative research questions, and part of our community has already started working on this exciting area of smart city OM. In this article, we shall discuss (i) the potential contribution that OM can make to the smart city movement, i.e., how OM research may help inform discussion and decision making in the public and private sectors. In particular, the core principle of making cities smarter is in line with the OM literature. We then discuss (ii) how and why SC operations can be an interesting topic that possibly expands the scope and depth of OM theory, through drawing links to the existing and new streams of OM literature. Finally, we present (iii) a few promising research directions and examples, in domains such as energy, transportation (mobility), and retail.
\end{abstract}

Key words: Smart City, Innovative Operations, Technology-Enabled Operations, Sustainability

History: The paper is accepted for publication in Manufacturing \& Service Operations Management

(M\&SOM) on July 12020.

\section{Smart Cities, or Smarter Cities?}

The urban population worldwide has been growing rapidly. The United Nations estimate that urban settlements account for 54\% of the world's population in 2016, and projects that the proportion will exceed $60 \%$ by 2030 [82]. More cities are being formed and existing ones are becoming bigger, denser and more complex. Rapid urbanization poses a number of grand challenges to cities around the world: from economic and social problems (e.g., income inequality, unemployment), planning problems (e.g., housing shortage, traffic congestion), to sustainable development (e.g., in energy and transportation), to education, health and crime problems associated with high population density. It is imperative for the public and private sectors to work together and look for solutions.

The smart city movement is the holistic approach to tackle these challenges by drawing on smart technologies and data analytics. Widespread availability and adoption of digital and networked technology makes possible a variety of innovative solutions to urban problems in domains such as mobility, energy, pollution, health, water, waste management, law enforcement, and so on. The business opportunities are enormous - the emerging sector of "urban tech" has drawn $\$ 44$ billion, 
or $22 \%$ of global venture capital investments, in 2017. This far exceeds other hot sectors such as pharma and biotech ( $\$ 16$ billion), and artificial intelligence ( $\$ 12$ billion) [29].

There is no surprise that numerous alternative definitions exist for a new industry buzzword such as "smart city." A few examples are provided below:

- IEEE [47]: A smart city brings together technology, government and society to enable the following characteristics: a smart economy, smart mobility, a smart environment, smart people, smart living, and smart governance."

- Cisco [26]: Cities that adopt scalable solutions that take advantage of information and communications technology to increase efficiencies, reduce costs, and enhance quality of life.

- IBM [46]: A city that makes optimal use of all the interconnected information available today in order to better understand and control its operations and optimize the use of limited resources.

- UK Department for Business, Innovation and Skills [81]: The [smart city] concept is not static: there is no absolute definition of a smart city, no end point, but rather a process, or series of steps, by which cities become more liveable and resilient and, hence, able to respond quicker to new challenges. Thus, a Smart City should enable every citizen to engage with all the services on offer, public as well as private, in a way best suited to his or her needs. It brings together hard infrastructure, social capital including local skills and community institutions, and (digital) technologies to fuel sustainable economic development and provide an attractive environment for all.

While there does not seem to be a consensus-based checklist of sufficient or necessary conditions for a given city to qualify as "smart," a few common themes echo among the alternative definitions, including: (smart and connected) technology, data and information, and efficiency and operations. Furthermore, it is evident from these definitions that the goal of the smart city movement is to make the urban environment smarter, i.e., continuously improving rather than moving toward a static goal. Some of the objectives that cities strive to improve include efficiency (in terms of cost and time), transparency, sustainability, governance, involvement (of citizens), safety, and health. As shall be evident from a few examples discussed later, many smart city problems fundamentally involve continuously improving multiple (possibly conflicting) objectives, by managing, informing and coordinating the actions of possibly decentralized agents. Such ideas have long been the core of the Operations Management (OM) discipline. In this paper, we shall discuss the connections between the smart city movement and the OM discipline, both in terms of how the OM community can contribute to the movement, and how the movement motivates interesting questions for us to advance OM research. In Section 2, we shall first provide the conceptual framework linking the core elements of a smart city with the opportunities they offer. In Section 3, we discuss some related 
work in other fields such as Transportation, Urban Planning, and Information Systems. Then, we shall discuss a few examples of smart-city-motivated research questions in OM, and how these can be interesting both from the (OM) theory and practice perspectives, in Section 4. Finally, Section 5 concludes the discussion.

\section{Core Elements and Opportunities}

The smart city movement is underpinned by the elements of technology, data, and operations. Systematic integration of these elements sets the foundation for innovative solutions to many urban problems and generates a number of opportunities to improve urban life. In this section, we shall discuss three opportunities of particular interest to the OM community, namely, transparency, efficiency, and involvement. Figure 1 illustrates the conceptual connections between the three core elements and opportunities.

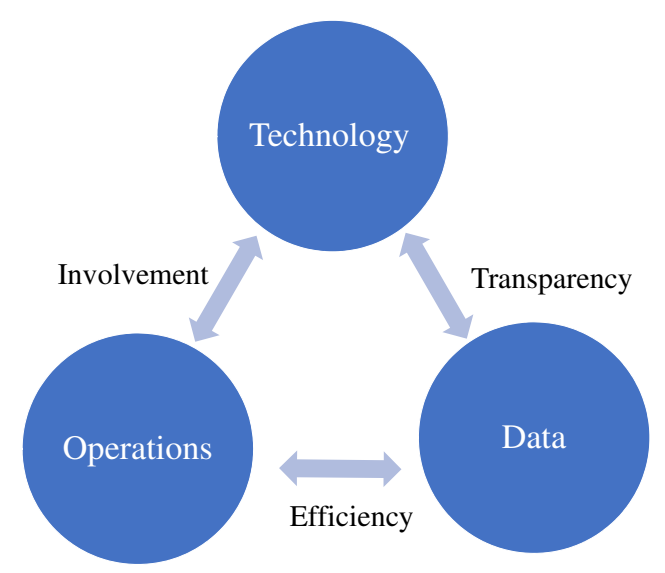

Figure 1 The Core Features of a Smart City

\subsection{Technology}

Perhaps the most visible feature of smart cities is the use of digital and connected technology. A smart city relies on a network of centralized and decentralized devices working and communicating with each other over the cloud, known as the Internet of Things (IoT). Many of these devices are sensors, which can be broadly defined as devices that collect data about their surroundings. These can range from more conventional touch, light or temperature sensors, to devices such as cell phones, CCTVs, smart watches, and smart (electricity, water or gas) meters that collect different forms of data. These sensors are networked via Wifi or cellular (and soon, 5G) networks and, in many cases, communicate in real time with data servers. The scale of the IoT is enormous and continues to grow rapidly. It is estimated that the number of connected devices used by smart cities worldwide would grow from 1.6 billion to 3.3 billion by 2018 [32]. 
Beyond data collection, many IoT devices serve additional functions as multi-way communication devices, computers (capable of running certain algorithms) and controllers (for other devices or appliances). For example, an Uber driver's smart phone collects data about his/her real-time location, uploads such data to the server, and communicates ride assignments and acceptance with the network. A home smart electricity meter could collect and upload electricity consumption data, and receive real-time pricing and weather information, based on which it could (working with a smart home controller) turn appliances on or off to reduce electricity costs.

Most IoT devices are relatively small in scale and seldom equipped with computing power to directly process data or run sophisticated algorithms on their own. With the development of cloud computing, intensive computations of data processing, analytics and decision algorithms can be run on the cloud, and the results communicated to the local devices. For instance, today's GPS navigation systems are capable of running route optimization algorithms on the cloud by taking real-time traffic information into account, improving over offline GPS devices in the past that performed such computations on board with stored map data that were only occasionally (in many cases, never) updated.

The fact that smart city solutions rely upon a decentralized network of independently and autonomously operating devices brings both challenges and opportunities. One interesting opportunity is the use of smart contracts. Based on blockchain technology, smart contracts enable decentralized agents on the IoT to engage in transactions that are contingent on events autonomously verifiable on public blockchain platforms (e.g., Ethereum). For instance, a homeowner (or its smart meter) can purchase solar power from its neighbor who owns a solar panel at a mutually-agreed price. Under a smart contract, payment can be completed contingent on delivery of power (i.e., power is fed from the solar panel system), as verified on the blockchain. Such a peer-to-peer trading model bypasses the intermediary (e.g., utility firm) and improves efficiency.

\subsection{Data}

Ubiquitous deployment of IoT devices in our urban environment leads to the collection of data in unprecedented volume, speed and scope. Sensors equipped on vehicles, for example, can keep track of vehicles' (and fleets') GPS location, speed, fuel consumption and other status in real time. The data is then uploaded to the cloud where it is stored and processed. Cisco estimates that, with the continued growth of the IoT, the amount of useful data generated by people, machines and things worldwide will grow to $85 \mathrm{ZB}\left(8.5 \times 10^{13} \mathrm{~GB}\right)$ in 2021, from $22 \mathrm{ZB}$ in 2016 [21]. The real-time generation of data enables the digital twin concept - On the IoT, it is possible to create a digital replica of each physical device, which can be monitored and analyzed digitally in real time. 
Supported by data supplied by the IoT, integrated models of the network of digital twins allows for visualization, experimentation, simulation and optimization of various operations before physical implementation.

In the IoT, data is collected and possessed by decentralized parties, e.g., city government, utility firms, public transit agencies, etc. As decisions and operations of these parties are naturally intertwined in the complex urban environment, there can be substantial economies of scope among their individually-possessed data sets, i.e., value can be created by sharing private data with other parties. In the public sector, more and more cities worldwide have embraced the open data movement by opening up their databases and requiring companies and organizations to do the same for their operations involving public interest. Open data refer to those that "anyone can freely access, use, modify, and share for any purpose (subject, at most, to requirements that preserve provenance and openness)" [63]. The Open Data Census [64] ranks 129 municipal areas in the US by the number and accessibility of open datasets, across multiple categories that include, e.g., crime, budget, transit, public spending, service requests, enforcement violations, etc. The top five cities in the 2017 rankings were Austin, San Francisco, Las Vegas, New York City, and Los Angeles, all of which have set up easy-to-access public repositories for their data. As we shall discuss in Section 2.4.1, the transparency enabled by open data initiatives generates important value to citizens and companies.

Unlike governments, the private sector is often less motivated to open their proprietary data for free. Yet, many firms in smart-city-related businesses, e.g., auto manufacturing, energy, and smart homes, increasingly recognize their proprietary data as valuable assets that can be monetized in the smart economy. That is, they recognize that data collected for certain primary proprietary purposes could be valuable to other companies or organizations, and possibly competitors, for other applications. For instance, an energy storage firm may deploy battery systems at individual homes, equipped with local frequency sensing capabilities. Such equipment collects real-time, decentralized frequency data that can be valuable and otherwise costly to collect for utility firms. Similar to the case of public data, there can be significant economies of scope in data collection and analytics, and strong opportunities exist for firms to improve their operations with access to data collected by others.

The data-as-a-service (DaaS) movement is closely related to the development of the softwareas-a-service (SaaS) business model. Similar to SaaS, DaaS is built upon cloud computing. As data sets grow in size, they are naturally stored and processed on the cloud rather than locally. On the cloud, data owners can provide on-demand access to other parties as a service. DaaS is 
particularly interesting in smart cities, as it allows data owners to monetize on data assets by selling access to other parties who use them for different purposes than they are originally collected for. In the private sector, some firms have taken the initiative to open their data for free (e.g., the Uber Movement project, where aggregated travel data from Uber has aided transportation planning in cities across the world), while many others have reservations about opening their data for free. Thus, DaaS could emerge as a parallel model to open data, and thereby incentivize more investments in collecting and maintaining data. In Section 3.3, we shall discuss an example of the DaaS model further in the context of smart parking platforms.

\subsection{Operations}

Most references to smart cities in the popular press may appear to focus on the engineering (technology) and data science (data) sides of the development. Yet, as we discussed in Section 1, the smart city movement fundamentally centers on the idea of making the city smarter. That is, with smart technology and the broad array of (often open) data as a backdrop, we seek ways to make the city work better, through better design, planning, and delivery of its services. As we discuss below, this process encompasses state-of-the-art OM methodology and insights, and thereby makes OM another important pillar of the smart city movement.

Van Mieghem [85] provides a historical overview of the OM literature. Much of the earlier literature, which Van Mieghem refers to as classic and original $O M$, took a mostly physical or engineering view, focusing on physical movement of items and people, allocation of physical resources, and their optimization. As the literature progressed, much of our attention has shifted toward the study of operations economics and empirical OM, where the focus is often on developing insights into how and why certain phenomena happen in operations and their managerial implications. Along these lines, we often look into how decision makers, each of whom having their (possibly misaligned) incentives, (possibly private) information, and behavioral characteristics, interact in operations.

Many challenges in the smart city movement naturally fit well with knowledge produced by these streams of OM research. Many smart city problems overlap with topics that OM researchers have studied extensively and possess domain knowledge, such as energy, transportation/mobility, supply chain/ logistics, and health. Within these domains, both the conventional engineering perspective and the contemporary economics point of view of OM are highly relevant. In smart cities, we are interested (among others) in improving movement, flows and distribution of people, vehicles, cargo, and equipment within the urban environment, as well as allocation of resources (e.g., service time slots, energy storage capacity, etc.) among stakeholders and components of the city. For many of these domains, the pertinent questions can be formulated as decision problems well suited for the 
classic OM approach. On the other hand, a variety of problems involve managing and coordinating resources and entities to work together, leading to various interesting phenomena well suited for operations economics research. We shall explore several examples of both throughout Section 4.

While the problem domains and some fundamental problem features overlap between contemporary smart city problems and the OM literature, what is novel in the smart city movement are the new possibilities enabled by the deployment of smart technology and availability of new data. First, the wealth of data collected through the IoT enables substantial opportunities to innovate city operations from a data-driven approach. The OM community's decision-oriented (prescriptive) approach to data (Simchi-Levi [74]), with a focus on improving efficiency, provides a strong complement to the statistical and data science approaches that often focus more on the visualization, problem/issue identification (descriptive) and forecasting (predictive) aspects. Second, aided by connected technologies, smart city operations often involve people, firms and entities that interact in a coordinated manner and in real time. This paves the way to innovative operations models that were not possible before, particularly ones that promote involvement of citizens and service users. These connections shall be further discussed in the next section.

\subsection{Opportunities}

2.4.1. Transparency. IoT technology and accessible (especially open) data enables transparency in city operations. Citizens, either directly or through third-party reports and apps, are able to keep track of operations and finances of the city transparently. This transparency empowers the public and ensures government accountability, and is often one of the primary motivations for developing smart city strategies in democratic countries. As the city becomes transparent and status of services can be accessed in real time, citizens and businesses are better able to make decisions regarding their activities, through which new efficiency and/or business value is created. This development is consistent with the OM community's drive to engage in the type of data-driven research linked to decision making (e.g., as advocated by Simchi-Levi [74]).

To illustrate how transparency spurs innovation and creates value, consider the example of Transport for London (TfL), the transport agency of the city. The open data initiative of TfL has stimulated an "app economy" that has created substantial direct value (estimated at $£ 15$ million to $£ 58$ million per year) by saving travelers’ transit times through more than 300 third-partydeveloped apps [44]. Furthermore, after finding success in London, some of the apps (e.g., the Citymapper public transit navigation app) are able to expand to other cities worldwide, further enhancing the economic value of the open data initiative.

While transparency is generally considered beneficial, it is inherently intertwined with the issue of privacy. As the IoT collects data in large volume and scope in cities, and as data becomes more 
openly accessible, concern for privacy naturally arises. Concurrently with this trend, regulations regarding data privacy are also evolving. In the EU, the General Data Protection Regulation (GDPR) recently came into effect in May 2018 [28]. GDPR stipulates that information regarding whether, how and why data is collected must be provided to data subjects (e.g., travelers whose commuting data is collected) whenever requested. It also grants data subjects the right to have their data erased. California has also recently passed a counterpart legislation, the California Consumer Privacy Act (CCPA) [61]. While legislations such as GDPR and CCPA offer new protection for personal privacy in the digital economy, they could significantly affect the deployment of IoT and smart city applications. One issue with the new regulations, for example, is that personal data must be anonymized and aggregated appropriately when shared with the public (see the discussion in [37], for example). Could the potential cost (both monetary and technical) of compliance favor large firms in the market at the expense of smaller players, including startups? This would be a valid question to investigate.

2.4.2. Efficiency. The unprecedented volume, scope, and speed of data becoming available in smart cities enables new opportunities to improve efficiency of city operations. As an example, consider Milton Keynes, a town in England that strives to develop into a smart city with the aid of its booming tech sector. One notable project of its "MK Smart" initiative is to improve household waste collection services by replacing household refuse bins with sensor-equipped, IoT-connected bins that communicate their real-time status (e.g., $60 \%$ full) to the server [78]. This data allows the municipality to optimize garbage collection routes in real time, instead of operating a fixed bi-weekly collection schedule. The potential benefits of this application are obvious: Households no longer need to worry about overflowing bins, as they are collected whenever they are full; while the municipality saves unnecessary collection when bins are not yet full. In the US, Baltimore [38] has engaged in a similar project.

Obviously, the first cut to improving efficiency in this problem is to improve how waste is collected, by mining data on individual bin status (i.e., waste generation rates), locations and availability of collection vehicles, and possibly traffic conditions, to determine cost-efficient schedules and routes. With access to such real-time data, it is possible to design and operate a much more dynamic, demand-responsive service than the current fixed-schedule service. For this problem, expertise of the OM community can be valuable in developing dynamic routing algorithms (which shall exhibit some similarities with inventory-routing problems), as well as in quantifying potential gains from incorporating different degrees of real-time information into routing and scheduling of collection. For example, what is the value of deploying smart bins only at a fraction of households? There is likely to be a trade-off between the (costs of) information and the potential 
efficiency improvements, that OM-trained researchers and practitioners are well-equipped to model and assess.

That is not the end of the story - In OM, we are equally concerned about the engineering and economic aspects of efficiency. The former refers to how to optimize a given system, or in this example, how to optimally route and schedule the collection service dynamically in real time. The latter, on the other hand, concerns the problem of managing and aligning incentives of stakeholders in the system to maximize system-wide performance. In the waste collection problem, we may recognize that users' service demand (waste generation rates) can actually be endogenous to the information possessed by the service provider. In particular, as the service provider has access to the real-time status of bins, households may (rationally) expect collection whenever or before bins are full. As a result, households may have less incentives to curb their rates of generating waste, e.g., by reducing recycling effort. If this does happen (which could be an interesting topic to study empirically), the side effect of increased waste generation could negate the gains from improved logistical efficiency. That is, the more appropriate proxy for efficiency should not just account for the costs and utilization of fleet management, but also the alignment of incentives of service users.

The natural solution to the potential incentive problem is usage-based pricing. In many municipalities (including Milton Keynes), waste collection service incurs a fixed periodic fee, e.g., included in the council tax paid monthly. To alleviate the potential incentive problem, one possible remedy is to introduce a collection charge per bin or per unit volume. In OM, the possible effect of different pricing schemes on households' response has been studied, albeit in a slightly different context. Belavina et al. [10] study the effect of grocery delivery pricing models on household food waste generation. They find that subscription-based pricing could incentivize households to shop more frequently, which reduces food spoilage and thus food waste. In the household waste collection context, the trade-offs will likely be different, but a similar modeling framework could be utilized to identify conditions under which different pricing schemes could be helpful in improving efficiency of the system and eliminating the side effects of waste generation.

2.4.3. Involvement. As discussed earlier, the availability of data of higher volume, velocity and quality enables better operations to improve efficiency. Next, we shall discuss how integrating smart and connected technology in operations can improve the involvement aspect of city services and create new value. In particular, smart and connected technology enables an important feedback loop in the design and delivery of city services, which enables bottom-up involvement of users in the planning and delivery of services and leads to interesting opportunities.

One form of bottom-up involvement is achieved through end-users feeding their usage or preference data to service operators with technology. This allows service offering to be catered to 
individual users' needs rather than aggregate forecasts, since service operators have access to individual-level user data in real time. As an example, consider Beeline, a transit startup in Singapore offering minibus services. It attempts to find the sweet spot between the economies of scale of operating fixed routes and the flexibility of on-demand service. Beeline's unique innovation is that its service routes are designed based on a bottom-up crowdsourcing process. This contrasts sharply with the conventional, top-down planning approach: first design the system (e.g., network of routes) at the strategic level based on long-term forecasts, then plan operations at the tactical level (e.g., timetabling with seasonal demand forecasts), and finally fine tune day-to-day operations (e.g., running buses). In Beeline's case, the crowdsourcing approach allows riders to propose routes using its mobile app, and those routes with sufficient demand will be added to its operating schedule. This innovative process, coupled with its platform-based business model, makes it a very interesting topic to study from the OM perspective. We shall discuss this further in Section 4.4.

Citizen science is a recent development of public participation in scientific research (e.g., Gura [40]), based on the crowdsourcing of research elements such as data collection and analysis, and in many cases, entire research projects. While citizen science has found success in domains such as conservation and astronomy with substantial amateur communities, the approach has also received much attention in city planning. Mueller et al. [60] discuss the use of citizen science in designing urban environments. Public involvement in data collection and analysis, coupled with economics principles such as open innovation, offer strong potential to improve public services. Glaeser et al. [36] study the problem of using open tournaments as an alternative to hiring consultants for city governments in the context of developing predictive analytics models. They find that such a crowdsourcing approach can be beneficial when the government has tolerance toward project risk and low-cost labor is abundant, and discuss the implementation of this approach by the City of Boston in crowdsourcing predictive algorithms to improve restaurant hygiene inspections.

Another key strategy to enhance bottom-up involvement is through the democratization of service delivery, i.e., end-users are utilized as assets or resources, on top of being consumers and information providers, in part of the service process. This is made possible through the booming sharing economy and online platforms (see Chen et al. [18] for an up-to-date review). These business models aim to create value through an intermediary role of matching entities that require a product or service with those who can offer it. In the city context, platforms such as Uber, Didi-Chuxing, Instacart and Doordash enable individual service providers to collectively compete in markets otherwise dominated by large-scale firms. The crowd-based nature of these business models not only improves efficiency of operations (e.g., in mobility and urban logistics), but can 
also possibly enhance welfare of independent workers under the right conditions (Benjaafar et al. $[13])$.

As digital technology enables involvement, a significant challenge is that the lack of access to technology could impede disadvantaged communities from benefiting from digitalization. The digital divide has been known as a major barrier against inclusive development (see, e.g., Kalkanci et al. [49]). In the urban context, the digital divide can be closely related to (racial and economic) segregation. For example, bike sharing systems often have low demand in economically-disadvantaged neighborhoods. Part of the reason is that low-income residents often have difficulty accessing technologies - it is found that only $66 \%$ and $43 \%$ of low-income people of color in Philadelphia, Chicago and New York City had smart phones and credit cards, respectively [59]. If geographical deployment of service is planned based on such (censored) demand data, less resources will be allocated to low-income neighborhoods, which further exacerbates such inequality. To tackle this viscious cycle, Capital Bikeshare launched an online crowdsourcing campaign to let potential users vote on new bike share station locations. While this program has helped uncover unfulfilled demand [65], it may not fully eliminate the potential negative impact of the digital divide, especially as the voices from disadvantaged neighborhoods could be underrepresented. As urban operations become increasingly entangled with digital technology, it is essential to account for potential biases arising from technology access to truly unlock the opportunity of involvement.

\section{Research in Other Disciplines}

The smart city movement is a multifaceted development that ideally should be studied with interdisciplinary lens. In this section, we briefly discuss some related research of interest in disciplines outside of OM.

\subsection{Transportation}

With substantial overlapping interests between the OM and transportation research communities, there is no surprise that a good number of recent works in the transportation literature are closely related to smart city OM. Besides common topical interests, the two communities also often employ overlapping methodologies, such as (mainly network and integer) optimization, equilibrium modeling, and queueing analysis. The divergence between the two streams of literature lies mainly in the differing perspectives - whereas OM researchers tend to take a prescriptive or predictive view for one or few key decision makers possibly interacting with others (e.g., a company interacting with strategic consumers or competitors), our transportation counterparts often take the point of view of the wider transportation system (e.g., the social optimal vs. user equilibrium concepts). 
A core consideration of the transportation literature is congestion under user-equilibrium travel choices. The simplest setting that exemplifies this concept is the morning commute problem (Vickrey [86]), which concerns a capacity-constrained bottleneck (e.g., a highway segment) that a set of commuters wish to pass through. Commuters decide their departure times by considering both the costs of congestion and possible deviation from their ideal arrival times (arriving early or late). The problem is to evaluate the equilibrium scheduling outcome of commuters, and the potential impact of measures such as pricing and capacity expansion (e.g., Daganzo and Garcia [22]). Yang et al. [88] consider the additional constraint of limited parking space, which further incentivizes commuters to make earlier trips.

In the smart city context, researchers have investigated possible technology-enabled measures to tackle congestion by analyzing extensions of the morning commute problem. Nie and Yin [62] study the use of tradable travel credits as an alternative to road pricing. The credit-based scheme can lead to more equitable outcomes, because commuters are endowed with an equal share of credits, unlike in the conventional road pricing case where initial endowment depends on wealth. Liu et al. [54] consider the use of expirable parking reservations as a measure to ease both travel congestion and competition for parking. Liu [53] investigate the morning commute problem with autonomous vehicles, in which the accessibility of parking spaces become less of a concern, as autonomous vehicles can park themselves after dropping off commuters.

From the problem setup, the morning commute problem is closely related to the literature of queueing games in OM, as queues are a natural way to model congestion. We shall discuss some of these connections in Section 4.2. In the transportation literature, the focus is usually on system performance, i.e., the level of congestion, cruising traffic for parking, etc., which both overlaps with and differs somewhat from the usual considerations in the queueing literature on utilization, waiting times, throughput, revenue (in cases of pricing), social welfare, etc. Given the wealth of knowledge in queueing theory and game theoretic analysis in the OM community, there are clearly opportunities to exchange ideas with the transportation literature.

\subsection{Urban Planning}

Primarily concerned with the development and management of the built environment, the urban planning community has been paying close attention to smart cities. Engin et al. [25] discuss the impact of digital technology on three levels of urban management, namely, framing the future, evidence-based planning decisions, and real-time management. Framing the future refers to understanding and envisioning how new technologies, such as artificial intelligence, blockchains, platform technologies, and virtual/augmented reality, can disrupt existing models and redefine how cities are run in the future. These developments drive the city's evolution in a bottom-up, rather than 
the traditional top-down, approach, and future cities can be understood as systems operating out of equilibrium instead of in equilibrium (Batty and Marshall [9]).

Evidence-based planning decisions refer to the use of empirical evidence, generated with the aid of digital data, to inform and scrutinize public decisions on infrastructure investment and land development. Engin et al. [25] suggest that an analytics-enabled planning could serve as an objective approach that helps overcome issues such as NIMBYism, where residents often have the tendency to block developments or projects affecting their neighborhoods. The goal in this line of work is to develop a data-driven "evidence base" for planning decision support. This could help address NIMBYism by informing residents of the experience and benefits of alternative solutions that have been implemented elsewhere [25]. Examples of analytics-enabled planning include the analysis of street network infrastructure using OpenStreetMap data [14], and the use of street view images and computer vision algorithms to measure visual quality of streets, which is otherwise difficult to measure objectively [89]. A related direction is to develop data-driven algorithmic tools for urban design, e.g., Seoul's use of genetic algorithms to optimize the layout of pedestrian-only superblocks [68].

The potential of real-time urban management is significantly broadened in recent years by the availability of a diverse range of real-time data. This enables the concept of "nowcasting," i.e., shortterm predictions of important metrics that inform immediate interventions. Naturally, some of the most promising applications relate to nowcasting for mobility management. For example, using convolutional neural networks, Aschwanden et al. [5] develops models to measure transportation mode share using satellite images. Zhong et al. [91] use smart card data to uncover mobility patterns in several major cities.

Interestingly, these three layers of urban management loosely resemble the OM concepts of strategic, tactical, and operational level decisions. The communities look at overlapping topics through different lenses - while OM research often focuses on the physics and economics aspects of smart city problems, the urban planning literature often pays closer attention to the social aspects. For example, on the topic of ridesharing platforms, while OM researchers are often interested in operational decisions such as pricing and matching, urban management researchers are often more interested in issues such as mobility of residents and access to employment opportunities. Another example is in urban design - while the operations community often investigate the design of service systems (e.g., location and capacity of bike sharing docks), the urban planning community may explore the design of the city layout itself (e.g., layouts of pedestrian blocks). There are clear opportunities for both communities to exchange ideas in terms of both modeling approaches and analytical/empirical methodologies. 


\subsection{Information Systems}

The development of a data-driven smart city ecosystem draws a close parallel with that of software platforms, which has been a core focus in the information systems (IS) community (e.g., Tiwana et al. [80]). Microsoft Windows is an example of a software platform, on which applications are developed. A smart city parallel is Predix [33], a cloud-based software and data platform for industrial IoT management. It can be thought of as an operating system for the IoT - it manages communication among the sensors and devices connected to the network, processes and integrates the data generated from these devices or sourced elsewhere (e.g., open data), and runs various data analytics and optimization applications.

In the IS literature, an important question is on consumers' and firms' preferences between proprietary platforms (e.g., Windows) and open source ones (e.g., Linux or Predix). While open platforms can help accelerate innovation (Boudreau [15]), they may not necessarily outcompete proprietary systems (Economides and Katsamakas [24]). August et al. [6] find that the choice between open source and proprietary strategies can interact with the firm's strategic choice on product quality. August et al. [7] compare the different open source licensing policies and their effects on both the software originator's and competiting contributors' decisions on their operations in the service market.

Open data initiatives are another aspect of smart cities that closely relate to the study of open source strategies. Like open source software, open data sources that permit independent contributors to build upon can both foster innovation and change the competition dynamics. It is of interest to explore the incentives and competitive forces behind firms' choice between open and proprietary data strategies. A key difference is that smart city firms considering open data usually treat data monetization as a potential second revenue source in addition to their primary operations (e.g., mobility services), unlike most software firms considering the impact of open source on their main revenue of selling software (either the platform itself or applications built upon it). To some extent, this relates to the consideration of service markets for software platforms by August et al. [7]. The opportunity for OM is to integrate more features of the operations business model into the choice between open and proprietary strategies.

\section{Some Research Directions}

In this section, we discuss a few promising research directions in the smart city space, and point out some research questions that the OM community is well positioned to answer. In each of these topics, one can identify interesting problem-based (how to solve problems), phenomenon-based (why phenomena happen), and/or insight-based (developing intuitive understanding of problems) research topics (Tang [77]). The set of examples is selected (in part) out of personal interest, and is 
not intended to be comprehensive. For more discussion of promising smart city problems for OM, readers may also refer to the recent discussion articles by Qi and Shen [67] and Hasija et al. [41].

\subsection{Smart Energy}

In New York Times best seller Hot, Flat, and Crowded: Why We Need a Green Revolution - And How It Can Renew America, Thomas Friedman describes his vision of electric vehicles (EVs) in the future [31, Chapter 11]:

"...... You decided to park at this ramp after it won a bidding contest against the parking ramp around the corner..... Because you will be sharing with [the owner] the money you earn from selling extra electrons back to the grid. The entire roof of the parking ramp consists of solar panels that create clean electrons, which are then sold to the batteries of all the cars on the ramp...... SoCalEd bought 5 kilowatt-hours off your car battery through the universal plug. This helped the utility meet its peak load demand and keep its load profile flat, while you and the garage owner both made money."

As adoption of EVs and renewable power continues to pick up, Friedman's smart energy vision is getting closer to reality with vehicle-to-grid ( $\mathrm{V} 2 \mathrm{G}$ ) technology. V2G enables EV batteries to store power for the grid to serve two main purposes. First, power storage can help flatten the daily load pattern and reduce the use of costly peaking generation. Second, V2G can provide ancillary service (e.g., frequency regulation or operating reserves) to stabilize the grid against short-term and stochastic fluctuations in demand and generation, especially as renewable (wind and solar) power with intermittent supply continues to displace fossil fuel generation sources. EV batteries are well suited for energy storage, since typical passenger cars are idle for over $90 \%$ of the day on average. That is, if the necessary EV supply equipment (EVSE) is available wherever and whenever the EV is idle, an EV can be available for energy storage for most of the day. The key challenges are to deploy sufficient infrastructure (EVSE with bi-directional inverters to allow two-way power flow), develop smart grid control systems that optimize charging and discharging in real time, and formulate business models that incentivize EV owners to make their cars available.

A few business models for V2G have been proposed and being tested in the industry. First, under time-of-use pricing, V2G allows EVs to profit from intra-day price differentials, i.e., by charging when electricity prices are low, and selling back to the grid when prices are high. While this model sounds straightforward, it poses some key OM challenges. In particular, the energy trading strategy must accommodate car users' travel schedules and needs. In OM terms, this corresponds to controlling the inventory of battery state of charge (SOC) under the constraint that travel needs must be satisfied. Zhang et al. [90] consider the V2G problem with intra-day energy trading for 
EVs in a car sharing system. Their stochastic optimization formulation, based on a time-space-SOC network flow framework, efficiently solves for the optimal acquisition of EVs, parking spaces and charging equipment for the car sharing fleet.

The second key business model is to trade capacity rather than energy through operating reserves or regulation markets, in which EVs are paid for providing back-up capacity over certain periods, during which power dispatch is required when called upon. This business model is particularly desirable for two reasons. First, the equilibrium prices for these ancillary services are generally higher compared with spot energy prices involved in the energy trading model [2]. Second, in ancillary services such as frequency regulation, the dispatch ratio, the ratio of actual energy dispatched to the contracted capacity, is often low [84]. Thus, participation in regulation is less likely to interfere with meeting travel needs. However, these markets operate at the transmission (wholesale) level of the grid, where participation requires a sizable volume (in the order of megawatts of capacity). Therefore, EVs must participate collaboratively as fleets (in hundreds or more) to harness the potential of the regulation model. Mak [57] studies the problem of a collaborative V2G platform that coordinates a pool of individually-owned EVs to participate in the regulation market, by providing pricing incentives to users for plugging in their EVs and bidding aggregate battery capacity in the frequency regulation market.

More generally, smart grid systems make it possible to coordinate small-scale and decentralized energy devices, including storage systems such as EVs or stationary batteries and distributed generation units (e.g., solar photovoltaics), to aggregate their capacities to jointly participate in the power markets as a single entity. Such coordinating systems are referred to as virtual power plants (VPPs), which serve as intermediaries between the decentralized assets and the power markets. Working as a two-sided platform, the challenge for the VPP is to manage the risks associated with uncertainties in both the power markets (e.g., regulation prices and signals) and operations of the decentralized assets (e.g., travel needs of EVs). This involves identifying the optimal strategy of trading in the power markets (and/or peer-to-peer trading) on the one hand, and compensating individual assets on the other. One very important question for VPPs is how to allocate power and energy (including storage) capacity among different operations such as frequency regulation, energy trading, spinning reserves, demand response, and so on. There are a number of confounding factors in this problem, such as the uncertain supply of renewable generation, availability of storage capacities (derived from travel needs of EVs, which can in turn be influenced through incentives), as well as the different requirements and prices of electricity markets (e.g., contracting on capacity versus energy, bidding cycles, etc.). The OM concepts of operational flexibility and financial hedging (e.g., Chod et al. [20]) could be helpful in formulating the optimal strategies in this problem. 


\subsection{Smart Parking}

Up to $30 \%$ of central business district traffic in major US cities can be attributed to vehicles cruising in search for curbside parking [71]. A major focus in the smart city movement is on developing smart parking solutions that leverage connected technologies. The starting point of smart parking solutions is often the concept of transparency and visibility through provision of real-time availability data. Besides helping drivers find available spaces, such data also serve as the basis for demand-based dynamic pricing. San Francisco installed individual sensors at 8,000 parking spaces in a pilot study (the SFpark project) of demand-based pricing in 2011-2013. Analyzing the pilot data, Feldman et al. [27] empirically find that such a dynamic pricing scheme can enhance welfare in congested areas, and suggest a simple three-tier, utilization-based pricing policy. Their proposal is consistent with the scheme implemented by the city: the parking fee in a block goes up by 25 cents per hour each when occupancy reaches the thresholds of $30 \%, 60 \%$ and $80 \%$ [70].

However, making real-time parking data public is difficult due to challenges in data acquisition and access. Occupancy data can be collected in real time through various means. For paid parking lots, entry gates and CCTV-based automatic number plate recognition (ANPR) systems can keep track of vehicle entry and departure. For on-street parking, data could be collected through devices such as CCTVs, parking sensors or smart street light sensors. These sensors can be costly to deploy and maintain. At the end of the SFpark pilot, the San Francisco city government found it too costly to maintain the parking sensors in working order, despite the (sunk) installation costs. Thus, the new pricing system it implements is not based on real-time availability data from sensors, but rather the predicted availability from real-time data from meters (tracking arrival times and purchased session lengths, but not actual departure times), based on models trained on previous occupancy data [70]. The UK town of Milton Keynes faces a similar challenge of developing statistical models that predict occupancy levels based on sensors at only a subset of parking spaces [17]. From the revenue management perspective, this poses an interesting challenge. Most revenue management models start from the assumption of limited, but known, inventory. To what extent do the insights carry over to the case where the inventory level (available parking spaces) is not known precisely, but only up to a certain confidence interval? This is similar in spirit to the issue of inventory inaccuracy (Raman et al. [69]) and supply chain visibility (e.g., with RFID investments, Lee and Özer [52]). Taking one step further, it is also of interest to investigate the problem of how many sensors to deploy (and where), balancing between the costs and the potential revenue (or some efficiency-based objective) that is optimized based on data acquired by the sensors. One issue of interest is the possible trade-off between revenue extraction and information acquisition - it could be optimal, for instance, to deploy (more) sensors at locations not deemed important from the 
revenue perspective, but could provide important information to optimize the overall performance of the parking network.

Beyond data collection, managing data ownership and access is also a key issue. For travelers and third-party app developers to draw a complete picture on parking availability, it is important for both public and private parking operators to make their data available. Yet, the private sector is often unmotivated to make their data open, as the benefits are often unclear to firms. This motivates two important and related questions for researchers to investigate, namely, the incentives for private operators to open access to their data and the potential value of developing a centralized platform to pool such data. To study incentives for private operators to share data transparently, it is important to investigate what can be achieved when both public and private parking data are available on a pooled platform. ParkMe is a data platform that partners with private and public parking operators to provide real-time parking data. It has been recently acquired by INRIX, a leading provider of real-time traffic information, and whose apps have been built in to certain Audi and Toyota vehicles [30]. This acquisition enables interesting possibilities, such as advance or real-time reservation of parking spots using in-car apps and dynamic pricing. In this case, ParkMe leverages its multi-functional platform to entice parking lots to share their data.

Under the emerging DaaS business model, it is interesting to investigate incentives for parking lots to share data with each other and/or to the public. Wu et al. [87] investigate the information sharing game between private parking operators, where they choose whether to share their demand forecasts with each other while engaging in price competition. They find that direct information sharing can be more beneficial than indirect collusion when forecasts are accurate. This research direction is closely related to the literature on delay information disclosure in queueing systems. Hassin [42] addressed similar questions for a system of two competing service providers (queues), one of whose queue length is unobservable by customers. The analysis concludes that, while the observable queue attracts higher market share in equilibrium, the unobservable queue can close the gap by disclosing real-time delay information. In a recent study, Singh et al. [75] consider the competition between two asymmetric service providers, and address the questions of whether one of the service providers should make the first move in disclosing delay information, and whether the other should respond. They find that information disclosure could be more beneficial for lowcapacity servers than for those with surplus capacity.

As the main medium for disclosure of occupancy (and delay, in queueing systems) information is through digital technology such as smart phone apps, it is conceivable that not all potential customers have real-time access to it. $\mathrm{Hu}$ et al. [45] consider the impact of delay information 
disclosure in a queueing system when only a certain fraction of customers have access to such information. Interestingly, they find that throughput and social welfare of the system can be maximized when the fraction of uninformed customers is strictly non-zero. Thus, getting everyone equally informed is not necessarily socially optimal. This phenomenon is similar in spirit to the informational Braess paradox in congestion games of traffic networks (Acemoglu et al. [1]), which states that travelers with access to real-time travel times and select travel routes accordingly (e.g., through navigation apps) can be worse off with such information in equilibrium.

While the literature on queueing systems focuses on the performances of servers under alternative information regimes, one key feature of parking problems is that parking lots are just one element of the wider transportation system. One important type of interaction between parking operations and the transportation system is the impact of parking availability on road congestion in the context of the morning commute problem (e.g., Yang et al. [88]). It is very interesting, for example, to investigate the interactions between occupancy information disclosure (and as a natural extension, reservation systems) of parking lots and broader transportation questions including congestion, mode choice and transit operations.

\subsection{Smart Retail and Urban Logistics}

Retailers with traditional emphasis on the physical and online channels alike have recognized the importance of smart omnichannel retail operations, especially in urban markets. The key battleground is the lucrative same-day delivery segment in urban markets (growing at $20 \%$ per year). For consumers, the possibility of ordering items online through mobile or smart home devices (projected to deliver $\$ 40$ billion of voice-controlled online sales by 2022 [83]), to be delivered within two to three hours, offers great appeal. For retailers, the key challenge is in fulfillment operations. Under tight delivery time windows, orders must be fulfilled from local distribution facilities close to consumers, rather than from centralized warehouses. The major bottleneck is in the last-mile delivery leg between these decentralized local facilities to consumers. Such operations are very difficult to manage due to the lack of scale economies, as opposed to delivery operations based in large-scale, centralized warehouses. New technologies have opened up novel smart operations solutions to the fulfillment problem, including crowdshipping, drones, and hyperloop. These innovations motivate interesting OM research problems, as we discuss below.

As discussed previously in Section 2, the smart city development enables operations models that involve consumers and individuals more directly in planning and operations of services. This philosophy is exemplified by urban operations under the sharing economy. Part of Amazon's solution to the last-mile problem is its launch of Amazon Flex, an Uber-like service that makes use of gig 
workers to deliver to customers. WalMart has experimented with a different crowdshipping scheme, in which it enlists its in-store customers to help deliver to online customers in their neighborhoods. Mak [56] studies a peer-to-peer (P2P) crowdshipping scheme, similar to WalMart's experiment, where fellow in-store shoppers can perform deliveries for online shoppers. This model has been considered difficult to implement due to logistical reasons (e.g., liability issues, insurance coverage for shopper-deliverers, etc.). The paper raises the question of whether the P2P concept merits more attention, by contrasting it against the more common business-to-consumer (B2C) sharing economy model where gig workers engage in delivery services as a job or business opportunity (e.g., Amazon Flex).

Drones are widely considered as a promising technology for certain delivery applications, such as where terrain conditions are poor and where roads are congested. Two modes of drone-based delivery logistics have been proposed by retail giants - Amazon plans to use drones for last-mile delivery of packages (weighing up to five pounds) to end consumers [51]; whereas JD.com plans to use heavy-duty drones (with payloads up to one ton) for transshipments between distribution centers and last-mile hubs [4], supplemented by ground-based last-mile delivery. In the operations literature, the logistics of air drones (and airborne vehicles more generally) in urban areas has seen rising interest. For example, in the health care context, Cho et al. [19] study the joint design of the trauma center and air ambulance network. Boutilier and Chan [16] study the problem of deploying drone-delivered automated external defibrillators in cities. Both studies employ location-queueing models formulated as (nonlinear) integer programs. While none of these directly explore the retail delivery context, the common operations challenges in these problems involve determining where to deploy airborne vehicles, subject to response- and travel-time constraints. In the retail context, these considerations could be further complicated by issues such as inventory placement (and pooling effects) and delivery routing.

The hyperloop concept is a grand vision for future transportation systems, advocated by highprofile entrepreneurs, including Elon Musk and Richard Branson. Through underground tunnel networks that pods and vehicles can traverse in vacuum, the technology potentially offers a highspeed, no-congestion, and low-emission alternative to road transportation. There are a variety of concepts under the hyperloop umbrella, both for passenger and freight [55], as well as long and short (e.g., intra-city) hauls. Magway, a London-based startup, is investing in an underground pipeline network that links locations across London to outer towns such as Milton Keynes, where major companies operate distribution hubs (e.g., Amazon). Freight shipments can be sent by maglevpowered pods through the vacuum pipelines, bypassing miles of congested roads, at headways (time gaps between successive pods) below 0.5 seconds [79]. 
If successfully deployed, both the drone and pipeline technologies could have game-changing impacts on urban distribution strategies of online retailers. Think of Amazon as an example: For its Prime Now (two-hour delivery) service, it is very difficult to fulfill orders from fulfillment centers located outside the city under the status quo. Instead, the retailer operates Prime Now hubs in the city, specifically catered for fast delivery service. With a denser distribution network design, these facilities do not achieve the same economies of scale as large-scale fulfillment centers. The variety of products available is also relatively limited, due to both space constraints and reduced inventory pooling effects (since each hub covers a smaller geographical demand base). Using drones or a freight hyperloop network, however, it is possible to fulfill time-sensitive orders from large-scale fulfillment centers outside the city, to last-mile consolidation hubs in the city. This could lead to more efficient distribution network designs, improved product variety and lower distribution costs, without jeopardizing service times.

These developments motivate interesting OM research questions. For example, how should the pipeline network be designed, especially within the city? A key point is that the pipeline network design must be informed by the operations of the last-mile delivery operations that runs upon it. That is, the pipeline network and the retailer(s)' distribution network should be, ideally, jointly optimized. Similarly, to design drone delivery systems in this context, it is important to not only model response time and possible congestion, but also the geographical placement and availability of inventory. All of these call for careful operations modeling, ideally informed by real data.

\subsection{Route Crowdsourcing in Public Transit}

Beeline, as introduced in Section 2.4, operates an interesting two-sided market platform model for the public transit business. On the one hand, it solicits route suggestions from individual users; on the other, it enlists private minibus operators to serve the selected routes. At the surface, the model shares a few similarities with ride sharing platforms with carpooling, e.g., Uberpool. Yet, a fundamental difference (and one that makes it interesting from the OM perspective) is that Beeline operates fixed routes, a feature absent in ride sharing, in attempt to enhance economies of scale in operations.

One interesting aspect of this problem is on understanding whether the rigidity of fixed routes is indeed at odds with the need for flexibility in on-demand services. In the manufacturing context, the OM concept of process flexibility suggests that a small degree of carefully-deployed flexibility can be almost as beneficial as full flexibility (Jordan and Graves [48]). Could the same be true for Beeline, that it could fulfill most on-demand orders by mixing and matching a number of (almost) fixed routes? This could be an interesting question to be answered from a data-driven approach. 
More generally, it is of interest to characterize the trade-off between service fit (i.e., how well the service fits customers' individual needs) and operating costs, along the spectrum of possible service configurations ranging from fully flexible and dynamic (e.g., taxis) to fully rigid (e.g., buses with fixed timetables and routes).

Another interesting problem is in determining which routes to run. Sim [73] details the algorithms that Beeline uses to form routes based on user submissions, built upon variants of the vehicle routing problem. However, under the two-sided platform structure, it is interesting to examine whether delegating part of the routing decisions to bus operators can help improve system efficiency. One approach to do this is through combinatorial auctions, where the platform lists the pick-up and drop-off points to be served, and bus providers can bid for different combinations (corresponding to routes) at different prices. The potential advantage of this approach is that bus operators, especially those who specialize in specific geographical areas, could possess private information and/or be more efficient in serving certain route combinations (cost synergies). By allowing bus operators to form and bid for their own routes, the platform can potentially elicit such information to improve efficiency. Such an approach has been implemented in the procurement of school meals in Chile. Through a structural estimation approach, Kim et al. [50] find that the combinatorial auction mechanism maintains high allocative efficiency and allows reasonable margins for bidders in the procurement of school meals. It is of interest to evaluate whether the same could hold for the application of bus routing.

\subsection{Autonomous Vehicles}

Autonomous vehicles (AVs) are an emerging technology that has drawn much intrigue. All major automakers are investing in self-driving systems, and many have integrated partial self-driving technology in their new models. It is widely believed that full self-driving technology will become reality in the foreseeable future. AVs will benefit smart cities in a number of aspects, if widely rolled out. First, AVs can communicate with each other and coordinate their maneuvers, potentially increasing the amount of traffic that the road network can accommodate. Mirzaeian et al. [58] use queueing models to investigate the potential increase in capacity on multi-lane highways, both with or without dedicated lanes for AVs. Besides increasing road capacity, the self-parking capabilities of AVs can also help ease the inconvenience and cruising traffic in searching for parking spaces, which can in turn influence travel choices and congestion levels (Liu [53]).

Second, the fact that AVs can operate on their own without drivers enables new operations models. Another interesting possibility is for AVs to be shared among multiple travelers, which overcomes the inefficiency of low utilization rates of individually-owed cars. Baron et al. [8] consider 
the adoption of AVs under a peer-to-peer sharing arrangement, in which a number of household share a fleet of AVs that autonomously reposition themselves to fulfill travel needs of travelers. Besides the peer-to-peer model, it is also widely discussed that AVs can operate in fleets under ridesharing platforms. Siddiq and Taylor [72] consider the impact of potential AV adoption on the competition between platforms, such as Uber and Lyft. Interestingly, the use of AVs in sharing platforms bridges the gap between ridesharing and (self-service) free-floating car sharing models (e.g., He et al. [43]). Thus, there is a significant scope to link the two lines of literature.

Considering the above possibilities, one important question to address is to design mechanisms for owning and operating AVs, especially under sharing arrangements. From the aforementioned discussion, the first possible sharing model is a $\mathrm{B} 2 \mathrm{C}$ car sharing model in which a firm operates the AV fleet and serves travelers on a pay-per-use basis. This corresponds to the servicization business model (Bellos et al. [11]). The second model is the Uber model, where a platform coordinates the operations of individually-owned AVs through pricing and matching travelers with vehicles. The third possible model is community-based peer-to-peer sharing, where travelers form independent groups (possibly based on location compatibility) to share AVs. In such a model, it is important to design appropriate mechanisms to share the costs of ownership and operations, especially as travelers naturally exhibit heterogeneity in travel preferences and needs.

Another potential impact of AV technology is in long-haul trucking. First, using autonomous trucks possibly relaxes operational constraints due to drivers (e.g., shift lengths, rest stops, limitations on home bases, etc.), and can help improve operational efficiency of inter-city freight transportation. One promising possibility is to design a hub-and-spoke trucking system based on a network of trucking hubs in major metro areas, where the hub-to-hub legs are operated by selfdriving technologies without drivers, and the local legs (spokes) are operated with local drivers. Both the design and operations of such a network could lead to interesting OM questions, both from the perspective of operational efficiency and welfare of drivers. The second major impact of AV technology in trucking is the potential cost-savings from platooning, i.e., the linking of multiple autonomous trucks in convoys to cut down fuel consumption (i.e., lower costs and emissions) by reducing air drag. While major trucking companies may have enough trucks to form platoons on their own, it is also interesting to investigate if smaller companies can also work collaboratively to form platoons and share cost savings. To answer this question, it is important to address both the operational (scheduling and routing) and economic (cost sharing) aspects of the problem. For instance, the fuel consumption heavily depends on the position of the truck in the platoon (e.g., the first truck in the platoon does not benefit from lower air drag), and the formation and positions of trucks could depend on both schedule and routing. With the OM community's collective expertise 
in domains such as scheduling, network optimization and cooperative game theory, we are well positioned to tackle these interesting questions.

\section{Conclusion}

$\mathrm{OM}$ is a rather unique discipline: one may consider it a business subject deeply rooted in engineering, or an engineering subject that is business oriented. The interface nature sometimes makes it difficult to position our value to the broader academic and practice community. In this article, we have discussed how the nature of OM places our discipline in a special role in tackling smart city problems, for which understanding of both the engineering and business aspects are critical. We provided a few examples of smart city problems and discussed some promising research directions motivated from each. As one recent challenge facing the OM community is how to maintain relevance (to practice) of our research (Tang [76]), the high-impact application domains in smart cities offer a promising avenue to new research problems.

As one may ponder whether smart city operations can become a new "hot topic" in OM, we reiterate that much of what we have discussed overlaps with several important themes of (especially, recent) interest in OM. These includes (environmental and social) sustainability (Agrawal et al. [3], Drake and Spinler [23], Kalkanci et al. [49]), cleantech (Plambeck [66]), innovative business models (Girotra and Netessine [35]), health care (Green [39]), innovative platforms (Chen et al. [18]), and sharing economy (Benjaafar and $\mathrm{Hu}[12]$ ), among others. That is, many of us who are already working in these areas do not need to change our research agendas to get started on smart city OM. In identifying and motivating interesting research questions from practice, the fact that many smart city applications involve either the public sector or startups could help, since they are often more open to dialogues and collaborations with academics. This could also help in eventual dissemination and implementation of research findings.

Throughout the paper, we have discussed potential research topics of different nature, such as problem-based research of tackling practical problems identified in industry, phenomena-based work of investigating how and why certain development has happened (or will happen) in practice, empirical work of gaining insights into existing data sets, etc. While these could all be promising and valuable, these scenarios all involve academia following practice. Given the technology-driven and startup-heavy nature of the smart city development, it will be even more exciting for academics to be more entrepreneurial and propose new ideas ahead of and leading practice. Smart city hackathons are quite frequently held around the world and they often give rise to innovative ideas (e.g., see [34] for hackathons involving smart lighting data in San Diego). Following the model of recent supply chain data competitions organized by MSOM and POMS societies, it will be interesting for OM academics to compete within and outside our community to generate new ideas. 


\section{References}

[1] D. Acemoglu, A. Makhdoumi, A. Malekian, and A. Ozdaglar. Informational Braess paradox: The effect of information on traffic congestion. Operations Research, 66(4):893-917, 2018.

[2] L. Agarwal, W. Peng, and L. Goel. Using EV battery packs for vehicle-to-grid applications: An economic analysis. In Innovative Smart Grid Technologies-Asia (ISGT Asia), pages 663-668. IEEE, 2014.

[3] V. Agrawal, A. Atasu, and L. N. Van Wassenhove. OM Forum - New opportunities for operations management research in sustainability. Manufacturing $\&$ Service Operations Management, 2018.

[4] Z. Aleem. The Chinese version of Amazon is already using drones to deliver stuff to customers, May 2017. URL https://www.vox.com/world/2017/5/26/15677522/jd-amazon-drones-china-bezos.

[5] G. D. Aschwanden, J. S. Wijnands, J. Thompson, K. A. Nice, H. Zhao, and M. Stevenson. Learning to walk: Modeling transportation mode choice distribution through neural networks. Environment and Planning B: Urban Analytics and City Science, page 2399808319862571, 2019.

[6] T. August, H. Shin, and T. I. Tunca. Licensing and competition for services in open source software. Information Systems Research, 24(4):1068-1086, 2013.

[7] T. August, H. Shin, and T. I. Tunca. Generating value through open source: Software service market regulation and licensing policy. Information Systems Research, 29(1):186-205, 2018.

[8] O. Baron, O. Berman, and M. Nourinejad. Introducing autonomous vehicles: Formulation and analysis. Working Paper, University of Toronto, 2018.

[9] M. Batty and S. Marshall. The origins of complexity theory in cities and planning. In Complexity theories of cities have come of age, pages 21-45. Springer, 2012.

[10] E. Belavina, K. Girotra, and A. Kabra. Online grocery retail: Revenue models and environmental impact. Management Science, 63(6):1781-1799, 2016.

[11] I. Bellos, M. Ferguson, and L. B. Toktay. The car sharing economy: Interaction of business model choice and product line design. Manufacturing \& Service Operations Management, 19(2):185-201, 2017.

[12] S. Benjaafar and M. Hu. Operations management in the age of the sharing economy: what is old and what is new? Manufacturing \& Service Operations Management, 22(1):93-101, 2020.

[13] S. Benjaafar, J.-Y. Ding, G. Kong, and T. Taylor. Labor welfare in on-demand service platforms. Working paper, University of Minnesota, 2018.

[14] G. Boeing. A multi-scale analysis of 27,000 urban street networks: Every US city, town, urbanized area, and Zillow neighborhood. Environment and Planning B: Urban Analytics and City Science, page $2399808318784595,2018$.

[15] K. Boudreau. Open platform strategies and innovation: Granting access vs. devolving control. Management Science, 56(10):1849-1872, 2010.

[16] J. J. Boutilier and T. C. Chan. Response time optimization for drone-delivered automated external defibrillators. Working Paper, University of Toronto, 2020. 
[17] British Telecom. Case study: Smart parking in Milton Keynes, 2014.

[18] Y.-J. Chen, T. Dai, C. G. Korpeoglu, E. Körpeoğlu, O. Sahin, C. S. Tang, and S. Xiao. Innovative online platforms: Research opportunities. Manufacturing \&3 Service Operations Management, 2019.

[19] S.-H. Cho, H. Jang, T. Lee, and J. Turner. Simultaneous location of trauma centers and helicopters for emergency medical service planning. Operations Research, 62(4):751-771, 2014.

[20] J. Chod, N. Rudi, and J. A. Van Mieghem. Operational flexibility and financial hedging: complements or substitutes? Management Science, 56(6):1030-1045, 2010.

[21] Cisco. Cisco Global Cloud Index: Forecast and methodology, 2016-2021 white Paper, 2018. URL https://www.cisco.com/c/en/us/solutions/collateral/service-provider/ global-cloud-index-gci/white-paper-c11-738085.html\#_Toc503317525.

[22] C. F. Daganzo and R. C. Garcia. A pareto improving strategy for the time-dependent morning commute problem. Transportation Science, 34(3):303-311, 2000.

[23] D. F. Drake and S. Spinler. OM Forum - Sustainable operations management: An enduring stream or a passing fancy? Manufacturing 83 Service Operations Management, 15(4):689-700, 2013.

[24] N. Economides and E. Katsamakas. Two-sided competition of proprietary vs. open source technology platforms and the implications for the software industry. Management Science, 52(7):1057-1071, 2006.

[25] Z. Engin, J. van Dijk, T. Lan, P. A. Longley, P. Treleaven, M. Batty, and A. Penn. Data-driven urban management: Mapping the landscape. Journal of Urban Management, 2019.

[26] G. Falconer and S. Mitchell. Smart city framework - A systematic process for enabling smart + connected communities, 2012. URL https://www.cisco.com/c/dam/en_us/about/ac79/docs/ps/motm/ Smart-City-Framework.pdf.

[27] P. Feldman, J. Li, and H.-T. Tsai. Welfare implications of congestion pricing: Evidence from SFpark. Working Paper, University of Michigan, 2017.

[28] Financial Times. Companies under strain from GDPR requests, 2018. URL https://www.ft.com/ content/31d9286a-7bac-11e8-8e67-1e1a0846c475.

[29] R. Florida. The rise of 'urban tech', 2018. URL https://www.citylab.com/life/2018/07/ the-rise-of-urban-tech/564653/.

[30] Forbes. Inrix's acquisition of ParkMe could be a game-changer for navigation, 2015. URL https://www.forbes.com/sites/lianeyvkoff/2015/09/10/ with-parkme-acquisition-inrix-will-tackle-pre-paid-reservation-based-parking/ \#c4dbf1e68ad5.

[31] T. L. Friedman. Hot, flat, and crowded 2.0: Why we need a green revolution-and how it can renew America. Picador, 2009.

[32] Gartner. Gartner says smart cities will use 1.6 billion connected things in 2016, 2015. URL https: //www.gartner.com/newsroom/id/3175418. 
[33] General Electric. Predix: The industrial internet platform, 2016. URL https://www.ge.com/uk/sites/ www.ge.com.uk/files/Predix-The-Industrial-Internet-Platform-Brief .pdf.

[34] General Electric. CityIQ innovation apps center, 2018. URL https://developer.currentbyge.com/ cityiq/innovation-apps-center.

[35] K. Girotra and S. Netessine. OM Forum - Business model innovation for sustainability. Manufacturing \& Service Operations Management, 15(4):537-544, 2013.

[36] E. L. Glaeser, A. Hillis, S. D. Kominers, and M. Luca. Crowdsourcing city government: Using tournaments to improve inspection accuracy. American Economic Review, 106(5):114-18, 2016.

[37] Google. Learning statistics with privacy, aided by the flip of a coin, 2014. URL https://security . googleblog.com/2014/10/learning-statistics-with-privacy-aided.html.

[38] Government Technology. Baltimore rolls out smart trash cans, 2018. URL http://www.govtech.com/ fs/automation/Baltimore-Rolls-Out-Smart-Trash-Cans.html.

[39] L. V. Green. OM Forum - The vital role of operations analysis in improving healthcare delivery. Manufacturing ES Service Operations Management, 14(4):488-494, 2012.

[40] T. Gura. Citizen science: amateur experts. Nature, 496(7444):259-261, 2013.

[41] S. Hasija, Z.-J. M. Shen, and C.-P. Teo. Smart city operations: Modeling challenges and opportunities. Manufacturing \& Service Operations Management, 2020.

[42] R. Hassin. On the advantage of being the first server. Management Science, 42(4):618-623, 1996.

[43] L. He, H.-Y. Mak, Y. Rong, and Z.-J. M. Shen. Service region design for urban electric vehicle sharing systems. Manufacturing \& Service Operations Management, 19(2):309-327, 2017.

[44] Hogge, Becky. Open data's impact: Transport for London - Get set, go!, 2016. URL http://odimpact . org/files/case-studies-transport-for-london.pdf.

[45] M. Hu, Y. Li, and J. Wang. Efficient ignorance: Information heterogeneity in a queue. Management Science, 64(6):2650-2671, 2018.

[46] IBM. IBM offers smarter city assessment tool to help cities prepare for challenges and opportunities of unprecedented urbanization, 2009. URL https://www-03.ibm.com/press/us/en/pressrelease/ 27791 .wss.

[47] IEEE. IEEE smart cities, 2018. URL https://www.ieee-pes.org/pes-communities/ ieee-smart-cities.

[48] W. C. Jordan and S. C. Graves. Principles on the benefits of manufacturing process flexibility. Management Science, 41(4):577-594, 1995.

[49] B. Kalkanci, M. Rahmani, and L. B. Toktay. The role of inclusive innovation in promoting social sustainability. Production and Operations Management, 28(12):2960-2982, 2019. 
[50] S. W. Kim, M. Olivares, and G. Y. Weintraub. Measuring the performance of large-scale combinatorial auctions: A structural estimation approach. Management Science, 60(5):1180-1201, 2014.

[51] F. Lardinois. A first look at Amazon's new delivery drone, 2019. URL https://techcrunch.com/ 2019/06/05/a-first-look-at-amazons-new-delivery-drone/.

[52] H. Lee and Ö. Özer. Unlocking the value of rfid. Production $\mathscr{E}$ Operations Management, 16(1):40-64, 2007.

[53] W. Liu. An equilibrium analysis of commuter parking in the era of autonomous vehicles. Transportation Research Part C: Emerging Technologies, 92:191-207, 2018.

[54] W. Liu, H. Yang, and Y. Yin. Expirable parking reservations for managing morning commute with parking space constraints. Transportation Research Part C: Emerging Technologies, 44:185-201, 2014.

[55] B. Lloyd. A New cargo brand built for an on-demand world, 2018. URL https://hyperloop-one. com/blog/new-cargo-brand-built-demand-world.

[56] H.-Y. Mak. Peer-to-peer crowdshipping as an omnichannel retail strategy. Working paper, University of Oxford, 2020.

[57] H.-Y. Mak. Collaborative V2G platform in the regulation market. Working paper, University of Oxford, 2020.

[58] N. Mirzaeian, S.-H. Cho, and A. A. Scheller-Wolf. A queueing model and analysis for autonomous vehicles on highways. Working Paper, Carnegie Mellon University, 2018.

[59] Mobility Lab. Bikeshare systems get more creative with equity issues, 2017. URL https:// mobilitylab.org/2017/09/21/bikeshare-systems-get-creative-equity-issues/.

[60] J. Mueller, H. Lu, A. Chirkin, B. Klein, and G. Schmitt. Citizen design science: A strategy for crowdcreative urban design. Cities, 72:181-188, 2018.

[61] New York Times. California passes sweeping law to protect online privacy, 2018. URL https://www. nytimes.com/2018/06/28/technology/california-online-privacy-law.html.

[62] Y. M. Nie and Y. Yin. Managing rush hour travel choices with tradable credit scheme. Transportation Research Part B: Methodological, 50:1-19, 2013.

[63] Open Definition. The Open Definition, 2018. URL https://opendefinition.org/.

[64] Open Knowledge International. US City Open Data Census, 2018. URL http://us-city.census. okfn.org/.

[65] J. Owen, C. Neita, and J. Froehlich. Crowdsourcing bikeshare transit planning: An empirical investigation of Washington D.C. and New York City. Working Paper, University of Maryland, 2015.

[66] E. L. Plambeck. OM Forum - Operations management challenges for some cleantech firms. Manufacturing $\mathcal{E}$ Service Operations Management, 15(4):527-536, 2013. 
[67] W. Qi and Z.-J. M. Shen. A smart-city scope of operations management. Production 83 Operations Management, 2018.

[68] S. J. Quan, J. Park, A. Economou, and S. Lee. Artificial intelligence-aided design: Smart Design for sustainable city development. Environment and Planning B: Urban Analytics and City Science, 46(8): 1581-1599, 2019.

[69] A. Raman, N. DeHoratius, and Z. Ton. The Achilles heel of supply chain management. Harvard Business Review, May 2001.

[70] San Francisco Municipal Transportation Agency. Sensor independent rate adjustments (SIRA) methodology and implementation plan, 2014. URL http://sfpark.org/wp-content/uploads/2014/05/ SIRA-methodology-and-implementation-plan_2014_05-14.pdf.

[71] D. C. Shoup. Cruising for parking. Transport Policy, 13(6):479-486, 2006.

[72] A. Siddiq and T. Taylor. Ride-hailing platforms: Competition and autonomous vehicles. Working Paper, University of California at Los Angeles, 2019.

[73] D. Sim. Open sourcing Beeline: How we do our routing?, 2017. URL https://blog.data.gov.sg/ open-sourcing-beeline-how-we-do-our-routing-ff7dd31675e2.

[74] D. Simchi-Levi. OM Forum - OM research: From problem-driven to data-driven research. Manufacturing ES Service Operations Management, 16(1):2-10, 2013.

[75] S. P. Singh, M. Delasay, and A. A. Scheller-Wolf. Evaluating the first-mover's advantage in announcing real-time delay information. Working Paper, University College London, 2017.

[76] C. S. Tang. OM Forum - Making OM research more relevant: "Why?" and "how?". Manufacturing 83 Service Operations Management, 18(2):178-183, 2015.

[77] C. S. Tang. OM Forum - Three simple approaches for young scholars to identify relevant and novel research topics in operations management. Manufacturing \& S Service Operations Management, 19(3): 338-346, 2017.

[78] The Guardian. Milton Keynes trials city-wide public 'internet of things' network, May 2014. URL https://www.theguardian.com/technology/2014/may/22/ milton-keynes-trials-public-internet-of-things-network.

[79] The Times. Shopping delivered by magnet in pipeline, 2020. URL https://www.thetimes.co.uk/ article/shopping-delivered-by-magnet-in-pipeline-gsn78thtc.

[80] A. Tiwana, B. Konsynski, and A. Bush. Platform evolution: Coevolution of platform architecture, governance, and environmental dynamics (research commentary). Information Systems Research, 21 (4):675-687, 2010 .

[81] UK Department for Business Innovation \& Skills. Smart cities: Background paper, 2013. URL https://assets.publishing.service.gov.uk/government/uploads/system/uploads/ attachment_data/file/246019/bis-13-1209-smart-cities-background-paper-digital.pdf. 
[82] United Nations. The world's cities in 2016, 2016. URL http://www.un.org/en/development/desa/ population/publications/pdf/urbanization/the_worlds_cities_in_2016_data_booklet.pdf.

[83] USA Today. Alexa, I need ... everything. Voice shopping sales could reach $\$ 40$ billion by 2022, 2018. URL https://eu.usatoday.com/story/money/2018/02/28/ alexa-need-everything-voice-shopping-becomes-common-sales-through-amazons-alexa-others-could-re $367426002 /$.

[84] S. I. Vagropoulos and A. G. Bakirtzis. Optimal bidding strategy for electric vehicle aggregators in electricity markets. IEEE Transactions on Power Systems, 28(4):4031-4041, 2013.

[85] J. A. Van Mieghem. OM Forum - Three Rs of operations management: research, relevance, and rewards. Manufacturing \&3 Service Operations Management, 15(1):2-5, 2013.

[86] W. S. Vickrey. Congestion theory and transport investment. The American Economic Review, 59(2): 251-260, 1969.

[87] Y. Wu, Q.-C. He, and X. Wang. Competitive spatial pricing for urban parking systems: Network structures and asymmetric information. Working paper, University of Wisconsin, Madison, 2018.

[88] H. Yang, W. Liu, X. Wang, and X. Zhang. On the morning commute problem with bottleneck congestion and parking space constraints. Transportation Research Part B: Methodological, 58:106-118, 2013.

[89] Y. Ye, W. Zeng, Q. Shen, X. Zhang, and Y. Lu. The visual quality of streets: A human-centred continuous measurement based on machine learning algorithms and street view images. Environment and Planning B: Urban Analytics and City Science, 46(8):1439-1457, 2019.

[90] Y. Zhang, M. Lu, and S. Shen. On the values of vehicle-to-grid selling in electric vehicle sharing. Working paper, University of Michigan, 2018.

[91] C. Zhong, M. Batty, E. Manley, J. Wang, Z. Wang, F. Chen, and G. Schmitt. Variability in regularity: Mining temporal mobility patterns in London, Singapore and Beijing using smart-card data. PloS one, 11(2):e0149222, 2016 